\title{
DÉSIGNIFIER AU PLURIEL: PARCOURS VERS LA DÉCHÉANCE
}

\author{
Richard-Laurent Barnett*
}

Ecrire, c'est avoir la passion de l'origine. ${ }^{1}$

Edmond Jabès

$\mathrm{M}$ achination fantasmatique en trois épisodes, "Cas de folie circulaire", premier texte publié par Michaux, date de 1922. Cette même année, la lecture de Lautréamont vient de déclencher "le besoin longtemps oublié d'écrire". 2 Or c'est précisément à une mise en scène de la difficulté du commencement que le lecteur est convié: la production du texte est tributaire de projections imaginaires concertées, mimant à la fois une amnésie volontaire et un excès irrépressible de mémoire. Chaque micro-récit postule la correspondance entre le présent d'une écriture, caractérisé par un état mental (identification, dysphasie, régression), une figure d'emprunt (Maldoror, une petite fille, un homme des cavernes), et une rhétorique empêtrée (parodie au second degré, théâtralisation d'une logorrhée, digression). Entre ces trois rêves d'annulation de la paternité et de l'histoire, le titre établit un lien ambigu: aussitôt qu'énoncée,

* Marygrove College.

1 JABÈS, E. Le Livre des questions. Paris: Gallimard, 1988. p. 360.

2 QR 13. Les références aux textes de Michaux seront indiquées par des abréviations, suivies du numéro de page. QR. Quelques Renseignements sur cinquante-neuf années d'existence. Les Cahiers de l'Herne, Paris, 1983, numéro spécial Henri Michaux; CFC. Cas de folie circulaire. Le Disque Vert, n. 5, p. 109-113, 1922; CA. Chronique de 1'Aiguilleur. Ecrits du Nord, n. 1, p. 25-29, 1922; RJ. Les Rêves et la jambe. Anvers: Ed. "Ça ira", 1923; A. Ailleurs. Paris: Gallimard, 1967; P. Passages. Paris: Gallimard, 1963; MM. Misérable Miracle. Paris: Gallimard, 1972; ER. Emergences-Résurgences. Genève: Skira, 1972; QJF. Qui je fus. Paris: NRF, 1927; FCD. Face à ce qui se dérobe. Paris: Gallimard, 1975; S. Saisir. Montpellier: Fata Morgana, 1979. Non paginé; AF. Affrontements. Paris: Gallimard, 1986. 
la folie, métaphore de l'originalité, ${ }^{3}$ prise dans "la sourcière du langage" (P 231), est en même temps déniée. Le discours littéraire simule un cas clinique décrit par Tibot (que Michaux a lu) comme une maladie quasi-psychologique.

Rien ne semble plus loin de la production ultérieure de Michaux que ces expérimentations bavardes, exhibant ironiquement leur ratage. ${ }^{4}$ Le troisième scénario, "Origine de la peinture", ébauche une parodie de régression dans la préhistoire; mêlant Rosny aîné à Homère et à Lautréamont, il semble tracer un nouveau cercle de l'intertextualité malheureuse. Pourtant, on peut y déchiffrer le projet d'une poétique qui, par la voie d'une digression narrative, se cherche: contredisant une élocution gonflée d'intertexte, l'invention donne à lire une parabole de l'immédiat et du trait, où le récit amphigourique dénonce sa propre incontinence, et où se réfléchit une esthétique naissante.

Le chapitre terminal de "Cas de folie circulaire" est d'ailleurs singulier à plus d'un titre. Texte-matrice, il inaugurait la série des 27 récits regroupés dans le premier recueil de Michaux, Fables des origines. Bien qu'il s'opposât, sur le plan rhétorique, au laconisme des autres "fables", il leur fournissait un cadre thématique, fictionnel et idéologique. Surtout, texte liminaire, il ouvre non seulement l'oeuvre écrite, mais aussi, d'une certaine façon, l'oeuvre peinte. Relevant d'une époque où son auteur prétendra qu'il "haïssait la peinture" (QR 14), cette rêverie étonnamment prémonitoire d'une pratique à venir proche de l'action painting illustre de manière saisissante le "désir de rupture" ${ }^{\mathrm{d}}$ 'une esthétique déjà insatisfaite par l'écrit.

\section{Un hypotexte surdéterminé}

La scène préhistorique expose le récit de Michaux à la forte pression intertextuelle d'un domaine codé, où se mêlent vulgate scientifique et vulgate idéologique. Selon Bataille, “la préhistoire n'est différente de l'histoire qu'en

3 Voir à ce sujet: FELMAN, S. La Folie et la chose littéraire. Paris: Seuil, 1978. p. $170-213$.

4 Le premier chapitre de "Cas de folie circulaire" est le seul à avoir fait le presque unique objet d'une étude: voir DÉPIERRE, A-M. Henri Michaux: "Il se croit Maldoror", figures et images. Revue d'Histoire Littéraire de la France, n. 5, p. 794-811, 1976.

5 Voir l'entretien avec JOUFFROY, A. Henri Michaux: Je me perds de façon très douloureuse. Arts, n. 746, 1959. 
raison de la pauvreté des documents qui la fondent". ${ }^{6}$ Comme le montrent Nadia Khouri et Marc Angenot, toute fiction préhistorique est nécessairement sous la dépendance d'un discours idéologico-scientifique, lui-même "largement imprégné par le modèle de la littérature romanesque". ${ }^{7}$

L'hypotexte d" "Origine de la peinture" est moins lisible par des signes mimétiques repérables que par la connivence culturelle inévitable qu'il instaure en 1922 entre l'auteur et un lecteur contemporain. Marquant un début de siècle travaillé, après bien d'autres époques, par le mythe des origines, La Guerre de feu de Rosny aîné propose en 1909 un modèle du roman préhistorique. La "dédicace" le situait d'emblée par rapport à une vraisemblance construite sur le modèle de la vérité historique, "aux temps où l'homme ne traçait encore aucune figure sur la pierre ni sur la corne, il y a peut-être cent mille ans". ${ }^{8}$ Il s'agira de produire ce qu'on pourrait appeler un "effet de caverne", de rendre crédible le procès de narration. Le mythe du feu est réinscrit dans le code de la quête romanesque, et le narrateur progresse prudemment dans une marge étroite, entre le trop de l'affabulation et le pas assez du savoir archéologique.

Cette émergence d'un nouveau type de fiction était, à son tour, étroitement liée à l'idéologie d'une réflexion anthropologique en particulier à celle des ouvrages de Lévy-Bruhl, tels que Les Fonctions mentales dans les sociétés inférieures (1910), ou La Mentalité primitive (1922). Rosny confirmait explicitement une telle contiguïté lorsqu'en 1923 il faisait paraître un essai intitulé Les Origines, la préhistoire, divulguant ses présupposés théoriques. Michaux lui-même semble s'être intéressé très tôt au Pithecanthropus, au chaînon manquant aux origines de l'homme: le "je" nettement autobiographique de Qui je fus dit avoir été, dès la fin de ses études chez les Jésuites, "vaguement tourné vers Haeckel” (QJF 11).

C'est pourtant un moment fondateur, dont ni la préhistoire ni le roman ne prétendent rendre compte, que Michaux, sur le mode ironique, se propose de représenter; le mythe de la naissance de la peinture rupestre désigne une scène primitive de la trace. Stratégie rouée: se sachant impuissante à imaginer un endeça de l'histoire, elle se confine avec ostentation dans la réduplication; un auteur particulièrement désireux d'échapper à la référence accuse le palimpseste, la singerie d'une fiction préhistorique.

6 BATAille, G. Oeuvres complètes. Paris: Gallimard, 1987. v. 10, p. 590.

7 KHOURI, N.; ANGENOT, M. Savoir et autorité: le discours de l'anthropologie préhistorique. Littérature, n. 107, p. 49-52, 1983.

8 ROSNY (AÎNÉ), J. H. La Guerre de feu. Paris: Plon, 1920. 
L'exergue en italique qui coiffe le récit (supprimé dans la version de Fables des origines) fonctionne à l'inverse de la dédicace de Rosny. Il esquisse, à travers un sujet extradiégétique, une figure auctoriale à double face; celle, obsédante, de l'écrivain qui "se surveille", "pion qui se voit élève"; 9 celle, rêvée, d'une identité barbare, antérieure à toute tradition:

\begin{abstract}
Il se figure être en préhistoire et son ignorance cyclique des noms d'Homère, de Virgile, de l'Egypte, de la Chine, est absolue et ne paraît guère être une feinte (...) Dans cet état, il ne reconnaît aucun nom propre connu, quoique son vocabulaire ne paraisse pas diminué autrement. Trait caractéristique: il fait autant de gestes avec le bras gauche qu'avec le bras droit et ses jambes sont également expressives. (CFC 112)
\end{abstract}

La prohistoire est ainsi présentée non comme un terrain déjà historicisé, mais comme le lieu d'une expérience-limite. En même temps, le texte est dénoncé comme un leurre; la pathologie simulée est significative: on y devine la mise en miroir d'un échec conscient, détourné en mystification. Selon une rhétorique qui chez Michaux sera codée, le "je" est tenu à distance par le "il". La recherche éperdue de la différence s'exténue en vaine gesticulation, et le projet de production d'un texte original est, au sens étymologique, ironique - il feint l'ignorance.

L'obsession de l'originalité ${ }^{10}$ prend de ce fait une forme paradoxale; elle se confronte à l'intertexte oraculaire et herméneutique d'un mutos universel, auquel participe un hypotexte exogène tel que l'Anthologie nègre, prédominant dans Fables des origines. A travers la thématique des origines, déjà centrale dans le recueil de contes africains compilés et publiés par Cendrars en 1921, Michaux s'inscrit ironiquement dans une mythologie contemporaine de la table rase, avatar de l'état original, lieu commun des avant-gardes auto-proclamées et

9 MICHAUX, H. Surréalisme. Le Disque Vert, n. 1, p. 83, 1925. Il faudrait annexer à cette problématique ce que Jean-François Lyotard disait des "espaces textuels" dans son Discours, Figure. Paris: Klincksieck, 1971; sans pour autant négliger cet autre "espace" si finement relevé par MARIN, L. Le récit est un piège. Paris: Minuit, 1978.

10 “...là réside, je pense, mon originalité, c'est que j'ai fouillé partout”, écrit Michaux dans une lettre à F. Hellens datée de novembre 1922 - citée dans HELLENS, F. Style et caractère. Bruxelles: La Renaissance du Livre, 1956. p. 156. 
du dadaïsme. ${ }^{11}$ Mais l'enjeu est aussi personnel: feindre d'ignorer le nom propre, c'est mimer une rupture avec le nom du Père, et c'est aussi manifester la volonté de conjurer les emprises les plus conscientes - lectures de jeunesse, passions pour le latin, le grec et l'art égyptien, "bricolage intellectuel" (QR 13). Volonté qui dénie elle-même son efficace, car entre le commentaire métalinguistique du préambule et le récit, le rapport sera d'ordre antiphrasique. Le premier revendique l'exclusion utopique d'un refoulé dont le retour investit le second; du désir de table rase, la parodie régressive sera une métaphore dérisoire.

\section{La main projective}

A l'opposé de La Guerre de feu, le "roman" sommaire de Michaux jette le trouble en l'espace d'un bref chapitre, dans le système romanesque repeint aux couleurs des premiers âges. D'abord récit de paroles, il juxtapose deux discours rapportés, dont le décalage marque, à la façon du discours psychotique, l'échec de la référence et de la communication. ${ }^{12}$

Le premier est une injonction ampoulée au style direct, où Brisgaieidiou ${ }^{13}$ - figure autoritaire du père - montre que loin de n'être bon qu'à la taille bifaciale du silex, il maîtrise les règles de l'éloquence cavernicole. Voici comment il appelle Ochtileou - en qui tout désigne la figure complémentaire d'un fils - à l'action contre un voleur de soleil: "Va, t'étant muni de pierres, par leurs formes propres au jet lointain, et dépouille cet homme, mu par un dessein caché qui, ayant grimpé sur la dernière branche du plus haut orme de la forêt, dérobe le soleil et l'ayant roulé devant lui jusqu'à l'aube parcourt une grande course. (CFC 113)"

A la façon de certains discours homériques, cette rêverie ascensionnelle de lutte contre les ténèbres est sans effet. Elle inaugure une série de ratés et de

11 Dans une "Conférence sur Dada", Tzara déclare en 1922: "Ce que nous voulons maintenant, c'est la spontanéité" et “...dada est un microbe vierge": TZARA, T. Oeuvres complètes. Paris: Flammarion, 1975. v. 1, p. 419-424.

12 Voir TODOROV, T. Les Genres du discours. Paris: Seuil, 1978. p. 78-85; ainsi que KRISTEVA, J. Distance et anti-représentation. Tel Quel, n. 76, p. 118-141, 1966; RIFFATERRE, M. Production du texte. Paris: Seuil, 1979; BARNETT, R-L. Tropismes sémiologiques. Littératures, Toulouse, n. 24, p. 111-127, 1999; BARNETT, R-L. Poétique des marges: lectures d'excentration. Paris: Presses Universitaires du Nouveau Monde, à paraître 2003 .

13 "Brise-caillou, brise-dieu", note MATTHIEU, J-C. Le Nom de l'autre. Europe, n. 95 , p. 698-699, 1987. 
distorsions - et d'abord, à la suite d'une ellipse narrative, l'errance filiale qui semble répondre, à travers les approximations d'un discours indirect. "à mission impossible, récit déceptif": "Ochtileou raconta au-delà des forêts les jambes pesantes qui collent à une terre colorée comme la nuque des oiseaux mâles, des empreintes profondes comme les racines des pins ou comme des sources chaudes - et le soleil entraîné par un fleuve retentissant." (CFC 113)

Les comparaisons multiples, de type homérique, déjà parodiées par Lautréamont, illustrent, par une recherche vaine des analogies, l'écart irremédiable entre l'immédiateté du vivre ou du voir et le mirage du dire. L'espace ainsi décrit, déjà creusé, aspirant vers le bas les pieds du marcheur, ressemble étrangement à celui du scripteur dont les traces laborieuses, cherchant l'expatriation, "collent" au préfabriqué du patrimoine linguistique, tellement sont "profondes" les "empreintes" anciennes du déterminisme intertextuel.

C'est là, sans transition d'aucune sorte, que commence véritablement l'action décalée de la fable, décharge et maculation dont Ochtileou est le sujet fugace. Le nom de ce peintre involontaire - Pollock du paléolithique, précurseur de la main projective et du tachisme - recèle la signature problématique de l'écrivain: où est-il Michaux? "Il jeta dans la caverne des becs, et des têtes d'animaux rares et une bête très grosse cachée dans une coquille épaisse et la terre molle, qui étant dedans, s'éparpilla sur les parois en masses inégales et diverses." (CPC 113) ${ }^{14}$

L'instantanéité accidentelle de l'événement n'est annoncée par aucune marque distinctive - syntaxique ou typographique. Elle se fond au contraire dans le réseau rhétorique qui, par un parallélisme de construction, assimile récit de paroles ("Ochtileou raconta/au delà des forêts/les jambes pesantes...") et récit d'actions ("Il jeta/dans la caverne/des becs"). De la même façon, la syntaxe déplace insidieusement le sujet grammatical, substituant la "terre molle", materia prima d'une alchimie spontanée, à Ochtileou, celui-ci, apparu fugitivement au

14 Cette "coquille épaisse" est une figure du cryptique et de l'intimité. Bachelard décrit ainsi l'imagination de la "vie repliée sur soi-même": "L'enseigne des objets habités pourrait être: Tout est coquille. Et l'être rêvant ferait écho: Tout m'est coquille. Je suis la matière molle qui vient se faire protéger dans toutes les formes dures, qui vient, dans l'intérieur de chaque objet, jouir de la conscience d'être protégé". BACHELARD, G. La Terre et les rêveries du repos. Paris: Corti, 1948. p. 18. C'est une intériorité que déplie, sortant de sa coquille, la "bête très grosse". Michaux écrit: “j”ai brisé la coquille/simple je sors du carcel de mon corps" Moments (Paris: Gallimard, 1973): 71. Consulter, sous ce rapport et toujours à profit, les commentaires pertinemment théoriques de RICHARD, J-P. Onze études sur la poésie moderne. Paris: Seuil, 1964; et, de même, la synthèse à la fois créatrice et manifestaire de PONGE, F. Le parti pris des choses. Paris: Gallimard, 1942. 
détour d'une phrase comme un acteur incident, est aussitôt dessaisi d'une action painting qui s'opère sans lui. Cet avènement sans préméditation de l'art pariétal rappelle l'aventure d'une toile de Twombly, que Barthes désigne du terme grec "tyché" (hasard); comme elle, il se manifeste par le double mouvement d'un "jeté" et d'un "dispersé". ${ }^{15}$

\title{
La fonction imaginogène
}

Le dernier "épisode" semble restituer au récit une cohérence qui lui manquait. Un alinéa introduit une nouvelle figure, féminine celle-là, confirmant le double sens de la scène primitive - parodie du mythe d'origine et esquisse de roman familial. A travers elle, surgit un nouveau sujet: à défaut de celui qui fait, celle qui regarde:

\begin{abstract}
Alors Isiriel, femme de Brisgaieidiou, très agréable certes, car couchée contre l'homme robuste, elle n'est pas inactive quant au mouvement de ses fesses, considérant avec rapidité la paroi de la caverne frissonna, ayant reconnu la virilité rouge, très puissante mais un peu tordue, d'un gorille incliné - et aussi ses yeux - et son geste. (CFC 113)
\end{abstract}

Isiriel - réminiscence phonique d'une Egypte prétendument oubliée apparaît comme un avatar sexualisé de l'Isis nervalienne ("Isis, la mère, alors se leva sur sa couche...). ${ }^{16}$ De même que le geste créateur était rabaissé en jet aléatoire sans trace et sans télos, d'un homo automaticus, de même le mystère isiaque est trivialisé, et la magie d'une apparition désublimée en vision libidinale.

15 Voir BARTHES, R. L'Obvie et l'obtus. Paris: Seuil, 1982. p. 167. Cette première peinture fait irrésistiblement penser à Pollock, mais aussi au groupe Cobra. Tous les éléments d'une animation spontanéiste (et non pas automatique) y sont, par avance, suggérés: la rapidité, la main projective, l'autonomie de la matière - et aussi la présence d'un regard (les yeux du gorille) qui émerge de la paroi, comme les yeux de l'entrelacs d'un tableau de Pollock. Voir à ce sujet: BUTOR, M.; SICARD, M. Alechinsky dans le texte. Paris: Galilée, 1984.

16 NERVAL, G. de. Oeuvres. Paris: Gallimard [La Pléiade], 1974. v. 1, p. 4. 
BARNETT, R-L. Désignifier au pluriel...

L'origine, dégradée en accident comme dans toutes les autres "fables", est introuvable - si ce n'est dans l'arbitraire phonique des signifiants, qui en retrace le dessin dérisoire: Isiriel/Brisgaieidiou/frissonna/gorille/virilité. C'est que l'antre protohistorique n'est pas pour Michaux le lieu fondateur décrit par Bataille, où c'est le génie ${ }^{17}$ de l'homo sapiens qui invente l'art mais plutôt un espace d'équivalences et d'analogies, où le regard d'Isiriel scrute l'altérité, obscur objet du désir, et l'identifie au connu. Cette reconnaissance de la trivialité, au sens étymologique, des différences (animalité/humanité, sexualité/érotisme) renvoie aux signes d'un déjà vu, à la façon dont la lecture identifie le texte, le réfère à une textualité autre. En même temps, reconnaître, c'est rapporter le visible au langage: le nom du gorille s'oppose à l'originalité innommable des "animaux rares". Sa figure anthropoïde, désignant avec précision les images ithyphalliques des cavernes peintes, ${ }^{18}$ inaugure le "cordon ombilical de la ressemblance", le "sacré de la première relation écrit-objet" (AF 83), un temps où l'image a été écriture, où "le premier essai de l'écriture a été une simple peinture". ${ }^{19}$ La Chine, à son tour, réinvestit obliquement un texte dont elle était rejetée: Michaux se passionne très tôt pour l'écriture chinoise; dans ses premiers idéogrammes, il décèle "la lisibilité primitive" (AF 77), idéal pictographique d'un protolangage.

Le frisson d'Isiriel marque la jouissance d'une transgression: il évoque cette "vacillation essentielle" où Lacan situe la pulsion scopique. ${ }^{20}$ L'iris de la spectatrice, perçant les ténèbres, interprète et produit un sens. Le rapport à l'image se confond avec l'origine du signe, il est théâtre mental, re-présentation du désir. Le fantasme d'Isiriel transpose un autre désir, celui du "triomphe par le ratage même" (ER 39), qu'accomplira plus tard la peinture à l'eau. Irreprésentable, clôturant le texte et lui imposant le silence, l'image du gorille qui se dépose sur la paroi obscure figure le raté de la narration; elle affirme contre un

17 Un chapitre de Lascaux ou la naissance de l'art s'intitule: "Le Rôle du génie": BATAILLE, G. Lascaux ou la naissance de l'art. Paris: Gallimard, 1979. v. 9, p. 26.

18 Voir BATAILlE, "Les Larmes d'Eros" dans ses Oeuvres complètes, v. 10, p. 580 - ainsi que Lascaux. Si la caverne peinte de Lascaux n'a été découverte qu'en 1940, celle d'Altamira le fut dès 1879 , et les travaux de l'abbé Breuil datent du début du siècle. Nombreuses sont les figures anthropoïdes et les images ithyphalliques. En particulier, l'homme de Hornos de la Pena a "une allure de singe", et son sexe est en érection (Lascaux, p. 86).

19 William Warburton (Essai sur les hiéroglyphes des Egyptiens), cité par TODOROV, T. Théories du symbole. Paris: Seuil, 1977. p. 273. L' acte de voir et de rereprésenter, entamé dans l'oeuvre todorovienne, est autrement exploré de façon d'autant plus nuancée par DÄLLENBACH, L. Le récit spéculaire. Paris: Seuil, 1977.

20 LACAN, J. Le Séminaire XI. Paris: Seuil, 1983. p. 79. A cet égard, les observations aussi perspicaces que percutantes de Jan Baetens pèsent: L'éthique et la contrainte. Louvain: Peeters, 1995. 
art de l'effet, la pulsion érotique, ou plus précisément une énergie érectile: la peinture "bande", écrit Barthes à propos de Réquichot (L'Obvie et l'obtus 45). Ainsi est rêvée une fonction auctoriale décentrée, dont les deux temps (production d'une forme allusive, vision intérieure), en rupture avec le conformisme des discours, brouillent la causalité et déplacent le sujet; elle est d'une part fonction projective, art de la rébellion filiale (le jet); d'autre part, "fonction imaginogène" (ER 80) de "celui qui avant les autres a vu" (ER 71), art de la délectation et de la jouissance maternelle (le frisson).

\section{L'ironie du rhéteur-traducteur}

Cette fable qui "raconte" l'avènement d'un signe pictural, dit aussi l'impuissance de la phrase: un rhéteur ironique creuse de négativité une odyssée elliptique, précipitée et sans objet - et compense la vacuité de l'epos par l'obscurité du mutos, mais aussi par l'excès de gesticulation verbale. Il porte à son paroxysme la tension entre les volutes et les boursouflures de la période, et la légèreté fuyante de l'anecdote. A l'action insituable, correspond une rhétorique doublement aliénée - surimpression d'un discours épique parodié et du style gauche de la traduction.

L'incipit, démenti de l'avant-texte, ancre ses procédures concertées dans une tradition qui récuse tout commencement, tout âge d'or et toute préhistoire: "Quand ils eurent dévoré le coeur, Brisgaieidiou suspendit la tête par les tendons enroulés trois fois" (CFC 112).

Début in medias res, passé simple réduisant au minimum la subjectivité, reprise du nombre sacré homérique, le "trois fois" déjà parodié par Lautréamont: ces stigmates d'un code narratif ancien prolongent Les Chants de Maldoror, "entreprise rhétorique contre la rhétorique". ${ }^{21}$ De même, dans la suite du texte, tics, clichés et redondances de l'appareil syntaxique miment l'exercice périlleux de la version grecque ou latine, où le malheureux traducteur-potache, soucieux de respecter une textualité originale, s'évertue à transposer, en des tournures archaïsantes et maladroites, un idiome étranger à sa propre langue. Le début

21 ROCHON, L. Lautréamont et le style homérique. Archives des Lettres Modernes, n. 123 , p. 22, 1971. Les procédés d'“Origine de la peinture" qui renvoient au style homérique et à la parodie de Lautréamont se recoupent évidemment avec ceux du premier chapitre "Il se croit Maldoror" de "Cas de folie circulaire": voir à ce sujet A. M. Dépierre. 
littéraire de Michaux renoue ainsi avec le début de l'adolescence: “Avec l'aide de son père, il s'intéresse au latin, belle langue qui le sépare des autres, le transplante; son premier départ. Aussi le premier effort continu qui lui plaise" (QR 12).

Mais la mimésis d' "Origine de la peinture" ne représente pas une échappée hors du conditionnement linguistique; elle retourne au contraire l'effet de primitivisme et d'étrangeté en effet de toge. Parodie de la parodie maldororienne, elle exhibe le labeur de l'élocution, surchargée de procédés tels que l'accumulation d'épithètes redondantes, de clichés ("un dessein cache", "une caverne obscure") et de coordonnants, les constructions impersonnelles ("Il est pénible pour l'homme de..."), la réticence ("Les panthères. . . ne désertent pas la piste fraîche"), l'emploi répétitif de participes passés composés ("ayant grimpé", "l'ayant roulé", "ayant reconnu"). La phrase, gauchie par une syntaxe retorse comme la virilité "un peu tordue" du "gorille incliné", pastiche la version qui impose à la langue héllenismes et latinismes: ordre inhabituel, parallélismes, appositions, répétitions maladroites, tournures bizarres. Un crescendo parodique signale cette perversion d'une rhétorique qui feint d'imiter, et tourne en dérision, à travers la culture classique, sa propre emphase: "Alors, Isiriel, (...) très agréable certes, car couchée contre l'homme robuste, elle n'est pas inactive quant au mouvement de ses fesses..."

Cette rhétorique de la traduction produit l'inverse du travestissement scaronnien: une amplification ironique enfle la syntaxe, tandis que le sujet même de la fable est progressivement trivialisé. La maladresse de la phrase ampoulée apparaît comme la manifestation d'un rapport détraqué au langage; le mythe n'est-il pas lui-même une "maladie du langage"? 22

La transformation dont l'hypotexte fait l'objet est de ce fait singulière. Elle brouille les traditions de la parodie, à la façon de ces "pratiques mixtes" où, selon G. Genette, "un même hypotexte peut à la fois (...) transformer un texte et en imiter un autre." ${ }^{23}$ L'exhibition de l'imitation stylistique, introduisant l'intertexte obsédant d'Homère et de Virgile dans le cycle du phrasé, porte un avertissement antiphrasique, en haine des écrivains, ces "ornementateurs vaniteux"; ${ }^{24}$ elle est démonstration outrancière de ce qui n'est plus possible, de l'envers de la modernité, à savoir la dérive d'une écriture dominée par le conformisme de la phrase.

22 MÜlLER, N. Leçons sur la science du langage. Paris: A. Durand et Pedone Lauriel, 1867. v. 2, p. 79.

23 GENETTE, G. Palimpsestes. Paris: Seuil, 1982. p. 39.

24 MICHAUX, H. Portrait d'homme. Mesures, n. 2, 1936, repris dans Les Cahiers de l'Herne, Henri Michaux, p. 337, 1983. 


\section{Feindre/peindre}

Prétendant rationaliser la bizarrerie du texte, l'énoncé terminal en accentue la dimension parodique: "Ainsi fut établi parmi les hommes combien l'image des choses est délectable".

Bien que restituant formellement l'archétexte canonique de la fable, cette clausule crée, à un endroit stratégique, une nouvelle surprise. L' "origine" fonde un consensus ("ainsi fut établi"), tandis que le code esthétique annoncé par le titre ("la peinture") se transforme en une image "délectable"; selon ce nouveau récit de genèse, le péché originel serait le voyeurisme - ou plutôt ce que Bossuet, après Saint-Augustin, appelle la concupiscence des yeux.

"La fiction, la déformation seule intéresse la littérature" (RJ 25), écrira Michaux un an plus tard. Simulation de fuite au plus loin du référentiel, feinte de rupture avec l'intertexte culturel, le récit fracturé d' "Origine de la peinture", par ses efforts imprévisibles de distorsion, met constamment à l'épreuve la compétence du lecteur, contraint de s'interroger: quelle est la cohérence de la fable? Quel est son vrai sujet? A-t-elle valeur d'apologue? Les isomorphismes qui la parcourent (parole/lumière/soleil/vision) sont masqués, par les ruptures diégétiques; mais les figures redondantes indiquent une isotopie de l'expression qui produit du sens là où l'on en attend le moins: dans la mise à nu, par l'emphase ironique, de l'arbitraire du langage-traduction, annonçant un renversement sémiologique - la contingence de l'icône. Le geste impulsif d'Ochtileou oppose la puissance spontanée d'un trait "sans intention particulière" (P 87) à la gaucherie cérémonieuse, anacoluthique du faussaire. La fabrication expansive du texte copie: traduisant la figure temporelle de la digression, diluant le narratif dans le descriptif, le descriptif dans la diversité conflictuelle des codes, elle épuise son effort de reproduction dans une théorique de l'indicible. L'image instantanée du "gorille", au contraire, signifie: participant du "monde des signes et des lignes" (ER 12) que Michaux verra dans la peinture chinoise, elle macule la paroi vierge du "phrasé même de la vie" (ER 9).

La mise en scène parabolique du jet et du frisson désigne le plaisir esthétique/érotique du simulacre. Mais elle ne se limite pas à dénier les pouvoirs de la narrativité, à (d)énoncer dans ce qui est lu, la réplique, à imaginer, médiatisé par l'ethos d'un descripteur, un modède différent de représentation - immédiateté d'une empreinte ou d'une anamorphose. Si elle s'affronte ouvertement à l'irréprésentable (le geste, le regard), c'est qu' elle rêve le possible d'une autre expressivité: la déhiscence d'une intériorité, aventure "surspontanée" (ER 40), exigeant "absence" et "aveuglement" afin d" "imprimer le mouvement en moi" 
(ER 109). Or c'est l'aventure du je-peintre, en rupture avec la réflexion du jeécrivain, que Michaux décrira en ces termes, cinquante ans plus tard, dans Emergences-Résurgences.

Si nous nous sommes attardés sur le détail textuel du troisième chapitre de "Cas de folie circulaire", ce n'est pas seulement parce que la critique l'a négligé. Son louvoiement ironique, circulation sinueuse d'un code à un autre, révèle une aspiration inverse: la recherche effrénée de l'entre-deux, du "no man's land", du court-circuit. Interrogation sur l'originalité, il appelle implicitement à une poétique en gestation, dont les orientations nouvelles (laconisme, fragmentation, création d'un idiolecte) vont se préciser dans Fables des origines, puis dans Qui je fus. Il marque aussi l'urgence d'une pratique hors de l'écriture, qui ne commencera à prendre forme qu'à partir de la peinture "tachiste" de 1925, mais qui inscrit déjà son origine, thématisée ici, dans l'inconscient d'un texte. La plaisante parodie d' "Origine de la peinture" cache une parabole sur l'art comme fulgurance et comme rapport électif.

\section{Ecriture et désir du geste}

Ce n'est pas la peinture, comme il le prétendra plus tard, que Michaux "haïssait", avant de découvrir en 1925 Klee, Ernst et Chirico. ${ }^{25}$ C'est l'art chargé de la mimésis, de la figuration. Une esthétique de l'incomplétude et du ratage, qui se voudra, comme la calligraphie chinoise, "ascèse de l'immédiat, de l'éclair (AF 99), commence à se formuler dès novembre 1922, deux mois après "Cas de folie circulaire", dans un "essai" paru sous la rubrique "Chronique de l'aiguilleur" des Ecrits $d u$ Nord. S'y énoncent, soit sous forme d'apologue, soit sous forme d'un discours enthymématique, des propositions elliptiques qui associent la modernité à l'agonie historique de la phrase et du phonocentrisme, et revendiquent contre la voix, le geste:

Tout le monde sait que le Théâtre meurt, la phrase parlée, la phrase gueulée, la phrase, l’Eloquence (....). Prédominance, développement

25 "Klee, puis Ernst, Chirico (...) Extrême surprise. Jusque-là, il haïssait la peinture et le fait même de peindre, 'comme s'il y avait pas encore assez de réalité', de cette abominable réalité, pensait-il. Encore vouloir la répéter, y revenir” (QR 14). 
prodigieux de l'image visuelle et prédominance sur celle-ci de l'IMAGE MIMIQUE, l'Intelligence mimique (...). Croyez-vous que l'expressionisme en peinture et en sculpture n'ait rien, rien du tout, rien à voir avec cette trompe du centre des images mimiques? (CA 26)

Les traits allusifs de l'apparition simiesque ("virilité", "yeux" et "geste") renvoient à cette identification de l'image à un mouvement, dont l'instantanéité est incompatible avec le caractère figé de l'écriture; ils préfigurent un geste de la peinture qui sera aussi peinture du geste: "J'aurais voulu dans un homme représenter le geste, partant de l'intérieur..."(S).

L'énergie du geste exprime spontanément une cénesthésie, donne sens à une materia prima, ductile et informe - "terre molle", origine plastique de la peinture qu'un alinéa de la "Chronique" nous présente à nouveau, en une fable-éclair, comme le donné brut de la sculpture: "L'Homme apercevait des blocs amorphes de terre molle, il a tripoté tant et tant que, maintenant, un passant distrait peut, de loin, prendre ça pour une femme nue. Sculpture!”(CA 27).

Cette glaise d'avant la signification est le parangon des matériaux, encre ou aquarelle, que Michaux choisira pour "faire du craché". ${ }^{26}$ Quant au "passant distrait", comme Isiriel il désigne la figure d'un spectateur fasciné par la ressemblance: son activité interprétative, liant une forme à un signifié, procédé du décryptage, de la lecture et de l'affabulation. Elle est menacée par le mimétisme. L'art, rencontre triviale, hasard objectif, sera contingent ou ne sera pas.

Les origines de la peinture et de la sculpture thématisent la recherche d'une sémiotique neuve. Michaux n'oppose pas encore, comme il le fera plus tard, l'écrit et le pictural. ${ }^{27} \mathrm{Il}$ les indexe au contraire sur la problématique commune de la modernité

26 A propos de ses encres noires, Michaux déclarait lors de son entretien avec A. Jouffroy: "Je fais du craché, du tout craché..." (Arts, n. 746, [1959]). Il écrivait en 1946, opposant la gouache à l'aquarelle: "La gouache résiste davantage à l'eau. Elle fait son petit mortier contre les évanescences qui la guettent. Elle tente de respecter les intentions de l'auteur, du respectable auteur!/Ne me convient pas" (P 111).

27 Cette opposition est particulièrement nette dans Emergences-Résurgences. Elle est cependant contredite par les échanges constants entre l'écrit et le peint, par la tendance idéographique d'une peinture que Zao Wou Ki n'hésite pas à qualifier d'“écrite" (Cahiers de l'Herne, Michaux, p. 385). Voir à ce sujet SIEBURTH, R. Ideograms: Proust/Michaux. L'Esprit Créateur, n. 36, p. 15-27, 1986. Il retrace l'itinéraire croisé de Michaux: “...adventurer in that semiotic no-man's land that exists somewhere between (or perhaps before) visual and verbal language" (p. 24). 
artistique, fascinée par le paradis perdu de la signifiance indivisée. "La spécialisation détruisit la tour de Babel": ainsi commence la préface de l'"essai littéraire et philosophique" de 1923, Les Rêves et la jambe. La "Chronique" voit dans l'art nouveau un mouvement général de dégagement, coupant court à une tradition décorative:

Mais nous, en littérature, en peinture "LA MAISON C'EST QUATRE MURS, UNE FENETRE, ET DU RESTE JE M'EN FOUS..."

Hygiène excellente!

Le cubisme, en peinture et sculpture, naît du même besoin actuel d'universalité et de simplicité que l'Esperanto. (CA 27)

Cette esthétique élémentaire désigne la modernité comme involution et comme ellipse. Plus rien ne serait original que le rêve d'un retour à l'originaire: plus rien ne serait possible que le fantasme d'une échappée en-deça du cycle historique de l'art - succession foisonnante de "tripotages", filiation confuse de ruptures et de greffes, d'écoles et de "bosses-procédés". Le "désert" métaphorise ironiquement l'utopie de ce degré zéro, topos réducteur d'une impossible modernité, elle-même déjà codée, qui mettrait entre parenthèses l'intertexte culturel:

Le XX Siècle-Art entre à la trappe, veut manger des racines, s'enfoncer dans le désert:

CUBISME, ART NEGRE

LITTERATURE ENFANTINE (CA 27)

Les débuts de Michaux participent de l'idéalisme surréaliste, référant l'écrit à l'authenticité antérieure d'un pathos, condamnant la littérature comme discours rapporté. Mais c'est en même temps une réflexion singulière qui 
prend alors naissance, et qui va se préciser à partir d'une pratique dédoublée, ${ }^{28}$ lorsque quelques années plus tard Michaux commencera à peindre. En 1922, il assigne encore à l'écriture, associée tantôt aux arts plastiques, tantôt à la musique, un pouvoir d'excéder ses propres limites, en précipitant le rythme d'un trajet plus libre, afin de l'accorder au "tic-tac plus rapide de représentations et d'émotions dans les arts" (CA 29). D'où les quelques explorations laconiques de Fables des origines, mimologiques et automatiques de Qui je fus, sagement éphémères.

Mais ces tentatives d'arracher le langage à sa pesanteur prédicative relèvent du désir de voix qu'est traditionnellement la poésie. A ce désir, comme à toute théâtralisation, le texte résistera - même et surtout lorsque sa forme semble particulièrement le rappeler: "Lectures sans voix. Prière aux comédiens de s'abstenir" (AF 114). Il sera au contraire travaillé par le désir du geste, privilégiant un rapport nécessairement insatisfait entre l'écriture et la peinture. L'espace textuel se voudra, comme la peinture de Michaux, "combat contre l'espace" (P 67), tendu entre deux pôles inverses: la destruction de la langue maternelle (par l'onomatopée, le minimalisme ou le cri) ou sa mise en oeuvre syntaxique-mimétique (par la chorégraphie de la "phrase événement") ${ }^{29}$ Ainsi l'exercice de pantomime liminaire d" "Origine de la peinture", auquel se livre un sujet qui "fait autant de gestes avec le bras gauche qu'avec le bras droit", n'est pas seulement gesticulation impuissante du corps, avant son engluement dans l'intertexte des mots; ce qu'elle mime paradoxalement, jusqu'à la désarticulation du pantin ("et ses jambes sont également expressives"), c'est l'aspiration de Michaux à la "stéréo-esthésie" (FCD 21), à une danse de l'écriture qui exprimerait spontanément, comme celle de l'Hindou, "les mouvements de la pensée (...) pour ne pas se désunir" (P 139). Cette préoccupation ne se démentira pas, d'une intégralité du corps propre, à retrouver par la combinaison de deux styles antinomiques: "l'ensemble droite-gauche (...) division à garder qui est aussi réunion” (FCD 66).

28 Voir HUBERT, R. R. Illustration and Double Talent. World Literature Today, n. 58, p. 209-215, 1984; ainsi que NICOLE, E. Les Lieux de la peinture et la linguistique de l'image. L'Esprit Créateur, n. 3, p. 37-50, 1986.

29 J'emprunte cette appellation à JENNY, L. La Phrase et l'expérience du temps. Poétique, n. 79, p. 283, 1989. 


\section{"La volonté, mort de l'art"}

Le geste pur, sans intention, projetant l'image d'un mouvement sur la paroi, inscrit l'intuition première d'une motilité, rêve d'une écriture gestuelle et, comme le rêve lui-même, "apparition du morceau d'homme sacrifié" (RJ 20). L'immédiateté érotique du gorille, rouge "dans un endroit de lui-même où est son feu", dessine, comme le "fantomisme" michaudien, "les traits du double" une "trame d'yeux" (P 93); la première peinture montre une imago du désir. Elle indique qu'un événement, au coeur de la rhétorique la plus éculée, traverse la phrase, - jet trouble, déroutant, qui échappe à l'adjectif, casse le rythme circulaire du trajet scriptural, le signale comme lieu de passage de l'ineffable. Mot-image d'un "être fluidique", le gorille annonce l'incomplétude du "fantôme intérieur" (P 92), l'intériorité des girafes de Misérable Miracle, "dessins formés par réflexion, non par copie" (MM 40). Il est le premier maillon, verbal, d'une longue descendance picturale.

Celle-ci commence avec la peinture tachiste des débuts, et surtout la "Tache" zoomorphe de 1926. Elle parcourt toute l'oeuvre: figures sur fond noir de 1937-38, "Tache" à l'encre de Chine rouge et noire de 1955 (reproduite dans ER 115), et ce livre exclusivement pictural de 1974, Par la voie des rythmes, "à michemin entre le hiéroglyphe et la bande dessinée", dont "l'inspiration générale se situe", selon Jean Onimus, "sous le signe de l'art des cavernes ou des images protohistoriques du val Camonica ou du Tassili”. ${ }^{30}$

"On s'étonne qu'une cavité souterraine ait été choisie", ${ }^{31}$ remarque Lacan à propos des peintures rupestres de la caverne d'Altamira, les premières découvertes. Michaux, lui, ne s'étonne pas: du noir, sa "boule de crystal", il voit "la vie sortir" (P 85). L'obscure paroi cavernicole est l'analogon du fond noir et de la nuit où se replie un imaginaire qui secrète, à l'écart de "la lumière mauvaise du soleil" (A 26), monstres et apparitions. Le support de la feuille noire manifeste "ce qui sort du néant, non d'une mère" (ER 22) - plus librement que le palimpseste de la page blanche. Le noir, "qui ramène au fondement, à l'origine" (ER 21), évoque la nuit des temps, l'espace primitif, sans dépôt, de la caverne, avant que le premier cryptogramme, encore "caché dans une coquille épaisse", ne marque

30 ONIMUS, J. Les Prouesses d'Henri Michaux: un livre sans écriture. Le Monde des Livres, n. 9190, p. 9-10, 1974.

31 LACAN, J. Le Séminaire VII. Paris: Seuil, 1986. p. 167. 
la coïncidence première du visible, du lisible et de l'illusion: 32 "Obscurité, antre d'où tout peut surgir, où il faut tout chercher". (ER 21)

"Dans le noir ce qu'il importe de connaître, et c'est dans la nuit que l'humanité s'est formée en son premier âge, et où elle a vécu son moyen âge". (ER26)

Traversant les ténèbres, un jet aveugle et sans signature invente l'art; l'inconscient du geste fulgurant d'Ochtileou préfigure l'épigraphe d'un texte de 1946, "En pensant au phénomène de la peinture": "La volonté, mort de l'art" (P 87). Est ainsi restituée, à une culture déchirée entre l'icône et la graphie, leur origine commune: la vitesse, l'énergie, l'élan du trait. Cinquante ans plus tard, Ochtileou devenu entre-temps Michaux peintre - il s'est, depuis, procuré de l'aquarelle - se décrit, avec une lucidité étonnante chez un ex-homme des cavernes, renouant sous le coup de la souffrance avec le geste originaire, se mettant à "projeter du hasard (...) de l'eau par giclées" (ER 31).

Eloge du "surspontané", le rêve préhistorique d'“Origine de la peinture" fait étalage de son antithèse, la contre-façon et la surcharge laborieuses. Tandis que la scène postule l'identité du lisible et du visible, la rhétorique joue la déchéance de leur séparation. "Pour retrouver un espace dégagé, où l'on se sente libre, il faut revenir aux peintures rupestres?" (P 69), se demande Michaux en 1945. Son oeuvre double, recherche éperdue de l'immédiat, ne cesse de s'interroger sur une réappropriation de l'origine. C'est une privation qu'un des tout premiers écrits commence par exhiber, entraînant déjà vers une pratique autre: le frisson d'Isiriel défie la facticité de l'écriture, appelle à en sortir. Michaux prédit ainsi, dès 1922, "un des plus étranges voyages en soi", le "déplacement des activités créatrices" (P 83), tentative de "déconditionnement" par la peinture:

Je devais sans doute rencontrer la peinture. La peinture est une base où on peut commencer à zéro. Support qui doit moins aux ancêtres. (ER 66)

32 L'exégèse michaudienne est pleinement en mouvement, ne cesse de se refaire, connaît, pour ainsi dire, un incessant renouvellement. Consulter, à titre d'exemple: FINTZ, C. Corps, écriture et spiritualité chez Michaux: quelques notes sur les derniers avant-textes d'une fin d'oeuvre. Littérature, n. 38, p. 115-127, 1998; LEBEL, J-J. La Danse neuronale. Magazine Littéraire, n. 364, p. 55-59, 1998; JACKSON, E. R. The Picture, the Poem, the Book: Henri Michaux's Three-Dimensional Creation. Harvard Library Bulletin, n. 6, p. 2940, 1995; ROGER, J. La Traversée des formes. Magazine Littéraire, n. 364, p. 48-50, 1998; et STAROBINSKI, J. Le Monde physionomique. Magazine Littéraire, n. 364, p. 52-54, 1998. 
BARNETT, R-L. Désignifier au pluriel...

Dans la peinture, le primitif, le primordial mieux se retrouve. (ER 14)

Voici, en somme, les enjeux d'un double mouvement - tantôt rupturé, tantôt déplacé, mouvement perpétuel et qui ne propage, il faut y succomber, qu'une voracité inamovible, la poursuite infinie et irrevocable de l' impossible. Rhétorique de la déchéance et curieuse dynamique, enfin. ${ }^{33}$

\title{
RESUMO
}

A poesia de Henri Michaux é submetida a um movimento inexorável de queda e ascenção: dos infernos do visual à esfera do textual, do mito das origens ao espectro do modernismo, da escavação e exumação ao ressuscitamento e à modulação, do hino da criação ao monturo da dissolução - e de volta ao princípio. Neste campo de batalha em que a linguagem e a arte estão soterradas, em que pintura e verso são unidos apenas para serem separados, apareceu uma renovada espécie de significante, um paradigma alternativo, eternamente à beira do desaparecimento, ainda que corajosamente equilibrada, de maneira a significar pela mesma pluralidade que ameaça seu ser.

Palavras-chave: Henri Michaux, poesia contemporânea, arte e literatura, intertextualidade, transtextualidade, dessignificação.

\begin{abstract}
The poetic of Henri Michaux is under-girded by a relentless movement: bellowing shifts from the realm of the visual to the sphere of the textual, from the myth of origins to the specter of modernism, from excavation and exhumation to resuscitation and modulation, from the song of creation to the knoll of annulation - and back again. On this

33 Etude copieusement subventionnée par la Fondation Treuhaft et par le Centre National de la Recherche Scientifique (Paris) - auxquels j'adresse ma plus vive reconnaissance. A ce témoignage il faudrait certes en annexer un autre: or cet essai de synthèse, eût-il été réalísé sans le long entretien méditatif dont Jean-Pierre Dupuy me fit l'amitié en mars 2002, à Paris. Trop rare privilège.
\end{abstract}


battlefield of language and art pitted, of paint and verse conjoined only to be severed there is birthed a newly-turned breed of signifier, an alternative paradigm, forever on the brink of eradication, yet stalwartly poised to mean by the very plurality that threatens its being.

Key-words: Michaux, contemporary poetry, art and literature, intertextuality, transtextuality, designification.

\section{RÉFÉRENCES}

BACHELARD, G. La Terre et les rêveries du repos. Paris: Corti, 1948. p. 18.

BAETENS, J. L'éthique de la contrainte. Louvain: Peeters, 1995.

BARNETT, R-L. Poétique des marges: lectures d'excentration. Paris: Presses Universitaires du Nouveau Monde, à paraître 2003.

. Tropismes sémiologiques. Littératures, Toulouse, n. 24, p. 111-127, 1999.

BARTHES, R. L'Obvie et l'obtus. Paris: Seuil, 1982. p. 167.

BATAILLE, G. Oeuvres complètes. Paris: Gallimard, 1987.

BUTOR, M.; SICARD, M. Alechinsky dans le texte. Paris: Galilée, 1984.

DÄLLENBACH, L. Le récit spéculaire. Paris: Seuil, 1977.

DÉPIERRE, A-M. Henri Michaux: "Il se croit Maldoror", figures et images. Revue d'Histoire Littéraire de la France, n. 5, p. 794-811, 1976.

FINTZ, C. Corps, écriture et spiritualité chez Michaux: quelques notes sur les derniers avant-textes d'une fin d'oeuvre. Littérature, n. 38, p. 115-127, 1998.

FELMAN, S. La Folie et la chose littéraire. Paris: Seuil, 1978. p. 170-213.

GENETTE, G. Palimpsestes. Paris: Seuil, 1982. p. 39.

HELLENS, F. Style et caractère. Bruxelles: La Renaissance du Livre, 1956. p. 156.

HUBERT, R. R. Illustration and Double Talent. World Literature Today, n. 58, p. 209$215,1984$.

JABÈS, E. Le Livre des questions. Paris: Gallimard, 1988. p. 360. 
BARNETT, R-L. Désignifier au pluriel...

JACKSON, E. R. The Picture, the Poem, the Book: Henri Michaux's Three-Dimensional Creation. Harvard Library Bulletin, n. 6, p. 29-40, 1995.

JENNY, L. La Phrase et l'expérience du temps. Poétique, n. 79, p. 283, 1989.

JOUFFROY, A. Henri Michaux: Je me perds de façon très douloureuse. Arts, n. 746, 1959.

KHOURI, N.; ANGENOT, M. Savoir et autorité: le discours de l'anthropologie préhistorique. Littérature, n. 107, 1983.

KRISTEVA, J. Distance et anti-représentation. Tel Quel, n. 76, p. 118-141, 1966.

LACAN, J. Le Séminaire VII. Paris: Seuil, 1967. p. 167.

. Le Séminaire XI. Paris: Seuil, 1983. p. 79.

LEBEL, J-J. La Danse neuronale. Magazine Littéraire, n. 364, p. 55-59, 1998.

LYOTARD, J-F. Discours, figure. Paris: Klincksieck, 1971.

MARIN, L. Le récit est un piège. Paris: Minuit, 1978.

MATTHIEU, J-C. Le Nom de l'autre. Europe, p. 698-699.

MICHAUX, H. Affrontements. Paris: Gallimard, 1985. Ailleurs. Paris: Gallimard, 1967.

. Cas de folie circulaire. Le Disque Vert, n. 5, p. 109-113, 1922.

. Chronique de l'Aiguilleur. Ecrits du Nord, n. 1, p. 25-29, 1922.

. Emergences-Résurgences. Genève: Skira, 1972.

Face à ce qui se dérobe. Paris: Gallimard, 1975.

. Les Rêves et la jambe. Anvers: Ed. "Ça ira”, 1923.

. Misérable Miracle. Paris: Gallimard, 1972.

Moments. Paris: Gallimard, 1973. p. 71.

Passages. Paris: Gallimard, 1963.

. Portrait d'homme. Mesures, n. 2, 1936. Repris dans Les Cahiers de l'Herne, Henri Michaux, p. 337, 1983. 
. Quelques renseignements sur cinquante-neuf années d'existence. Les Cahiers de l'Herne, Paris, 1983. Numéro spécial Henri Michaux.

Qui je fus. Paris: NRF, 1927.

. Saisir. Montpellier: Fata Morgana, 1979.

Surréalisme. Le Disque Vert, n. 1, p. 83, 1925.

MÜLLER, N. Leçons sur la science du langage. Paris: A. Durand et Pedone Lauriel, 1867. v. 2, p. 79.

NERVAL, G. de. Oeuvres. Paris: Gallimard, 1974. v. 1, p. 4.

NICOLE, E. Les Lieux de la peinture et la linguistique de l'image. L'Esprit Créateur, n. 36, p. 37-50, 1986.

ONIMUS, J. Les Promesses d'Henri Michaux: un livre sans écriture. Le Monde des Livres, n. 9190, p. 9-10, 1974.

PONGE, F. Le parti pris des choses. Paris: Gallimard, 1942.

RICHARD, J-P. Onze études sur la poésie moderne. Paris: Seuil, 1964.

RIFFATERRE, M. Production du texte. Paris: Seuil, 1979.

ROCHON, L. Lautréamont et le style homérique. Archives des Lettres Modernes, n. 123, p. 22, 1971.

ROGER, J. La Traversée des formes. Magazine Littéraire, n. 364, p. 48-50, 1998.

ROSNY (AÎNÉ), J. H. La Guerre de feu. Paris: Plon, 1920.

SIEBURTH, R. Ideograms: Proust/Michaux. L'Esprit Créateur, n. 3, p. 15-27, 1986.

STAROBINSKI, J. Le Monde physionomique. Magazine Littéraire, n. 364, p. 52-54, 1998.

TODOROV, T. Les Genres du discours. Paris: Seuil, 1978. p. 78-85.

Théories du symbole. Paris: Seuil, 1977. p. 273.

TZARA, T. Oeuvres complètes. Paris: Flammarion, 1975. v. 1, p. 419-424.

WOU KI, Z. Michaux. Les Cahiers de L'Herne, p. 385, 1983. Numéro spécial Henri Michaux. 